\title{
La incertidumbre de la democracia
}

\section{Josep M. Colomer *}

La democracia no necesariamente produce buenos resultados, es decir, decisiones socialmente eficientes, leyes y políticas públicas justas y actos administrativos eficaces. Por el contrario, está en la esencia de la democracia que sus decisiones sean inciertas y varien en el tiempo y que, por tanto, no correspondan a ningún conjunto concreto de valores que pueda considerarse intrínsecamente "bueno" o "superior". La incertidumbre de los resultados de la democracia y el relativismo de valores con que pueden ser juzgados corresponden precisamente a la certidumbre y la equidad de sus reglas de decisión. Las reglas democráticas de toma de decisiones pueden ser consideradas equitativas y tienden a durar en la medida en que garantizan la incertidumbre y el relativismo de las decisiones.

Estas características de la democracia -certidumbre de las reglas, incertidumbre de los resultados- la distinguen de modo fundamental de la dictadura y son la clave para que la democracia pueda ser ampliamente aceptada en la sociedad. La reciente expansión de las formas de gobierno democráticas a numerosas y variadas sociedades de diversas partes del mundo -y en particular de Europa central y oriental-, así como las expectativas de su consolidación, no se apoyan tanto en la bondad de sus resultados como en esa incertidumbre esencial. Pero la democracia es incierta también en otro sentido: su consecución nunca es segura, ni siquiera bajo las más favorables condiciones, ya que la democracia concebida como un procedimiento de decisión colectiva sólo puede ser resultado de un acuerdo que depende de las voluntades, las estrategias y las decisiones humanas y no de cualquier imaginaria ley histórica o determinación estructural.

\section{La democracia como resultado de decisiones estratégicas}

Los procesos de democratización iniciados a finales de los años ochenta han refutado el determinismo "estructural" con que el cambio de régimen había sido ampliamente analizado en las ciencias sociales a mediados de siglo. Ni en la Unión Soviética ni en ninguno de los demás países de socialismo autoritario de Europa central y oriental que han experimentado procesos de democratización existían los requisitos socio-económicos o culturales de la democracia que se habían postulado tradicionalmente -básicamente, una economía capitalista, una expansión de las clases medias y una amplia difusión de valores liberales. Parece, por tanto, lógico que el análisis de las condiciones de la democratización se haya acabado de decantar en los últimos años hacia las decisiones de los actores políticos y sus interacciones.

Para comprender mejor este cambio de orientación analítica, puede ser conveniente tener en cuenta cierta evolución del propio enfoque "estructural". La relación entre el desarrollo socioeconómico y la democracia política se había establecido tradicionalmente en unos términos bastante deterministas y unilaterales. La cadena causal establecida desde las estructuras sociales hacia la política implícitamente "condenaba" a los paises atrasados y pobres a sufrir regímenes autoritarios. Pero, a lo largo del tiempo, esta línea de causalidad fue alterada de diver- 
sos modos por algunos científicos sociales para que permitiera explicar diversas observaciones contractuales. Así, el desarrollo socio-económico fue presentado en algunos momentos como una condición necesaria, pero no suficiente para la democracia, dado que ciertos regímenes autoritarios aparecían como compatibles con el crecimiento económico o incluso como promotores de éste. De un modo similar, el desarrollo socio-económico fue presentado en otros momentos como una condición suficiente, pero no necesaria para la democracia, con objeto de explicar la larga supervivencia de regímenes democráticos en algunos países pobres y descolonizados.

Como consecuencia de estas reformulaciones, el postulado inicial que vinculaba el desarrollo socio-económico a la democratización política acabó perdiendo una gran parte de su atractivo: el desarrollo ya no era considerado una condición necesaria a la democracia -dado que algunos regímenes burocráticos-militares de América Latina y varios de los "tigres" asiáticos combinaban el autoritarismo con el crecimiento económico-, ni como una condición suficiente dado que varios países pobres, especialmente pertenecientes a la Commonwealth británica, la mayor parte de América Latina desde los años ochenta, así como muchos países de Europa oriental previamente dominados por los partidos comunistas desde los años noventa, sostenían regímenes democráticos.

Algunas tentativas recientes de establecer relaciones más sofisticadas entre las condiciones socio-económicas y las formas políticas a diferentes niveles de desarrollo económico sólo han hallado impactos pequeños y variables (una revisión reciente de este enfoque, en parte autocrítica, puede encontrarse en LIPSET, SEONG y TORRES, 1993; para una discusión más amplia, COLOmER, 1994). Aparentemente, el desarrollo económico puede crear condiciones favorables para que los individuos y los grupos deseen la democracia, luchen por ella y negocien su establecimiento. Pero de hecho la caída de las dictaduras también puede ser preparada o precipitada por sus fracasos sociales y económicos -incluida la incapacidad de los regímenes de socialismo autoritario de cumplir sus promesas de bienestar material e igualdad social. Así pues, si tanto los éxitos como los fracasos socio-económicos aparecen relacionados con el cambio de régimen político, parece conveniente aceptar que éste no puede ser explicado de un modo completamente satisfactorio por ninguno de aquellos, sino más bien que los resultados políticos deben ser conectados más explícitamente con las acciones políticas- es decir, las preferencias, las estrategias y las decisiones de los actores políticos. Mientras que en el enfoque "estructural", la política era considerada una variable dependiente de las variables socio-económicas y culturales, en el enfoque "estratégico", político, se tiende a subrayar más la influencia opuesta de la política sobre la economía, el establecimiento de lazos sociales y la difusión de valores culturales.
El énfasis en la indeterminación en las relaciones entre las estructuras socio-económicas y las estructuras (o regímenes) políticos no significa que las formas de la situación inicial autoritaria no influyan sobre la vía de cambio. Obviamente, un cierto grado de complejidad social o de pluralismo cultural es necesario para que se formen actores diferenciados que puedan entrar en interacción. De otro modo, en una sociedad altamente homogénea o incluso en una sociedad muy simplemente polarizada, las preferencias de régimen político tienden a ser también simples o frontalmente incompatibles, de modo que sólo una alternativa puede ser impuesta por el actor poderoso correspondiente. Los modelos clásicos de "revolución" o "guerra civil" pueden dar cuenta en tales casos del cambio de régimen, el cual puede aparecer como un reflejo bastante directo de las estructuras sociales.

Pero en sociedades más complejas y plurales, las mismas estructuras socio-económicas permiten diferentes resultados, bien de conflicto bien de estabilidad, bien autoritarios bien democráticos, producidos por diferentes vías de cambio. El cambio político no está garantizado ni siquiera cuando se cumplen muchas de las supuestas condiciones favorables, y aún menos cierta es la vía de cambio que seguirá un país concreto a partir de un acontecimiento crítico, dado que las vías y los resultados dependen directamente de las decisiones de los actores.

Las condiciones básicas para producir un resultado colectivo en un proceso de cambio de régimen están vinculadas a la fuerza y la iniciativa de los diversos actores políticos. Como ya he elaborado en otros lugares, las transiciones pactadas requieren ciertas condiciones estratégicas, entre ellas la ausencia o debilidad de los actores "maximalistas" -es decir, de aquellos que prefieren las alternativas políticas "extremas" a las intermedias, con preferencias de tipo "antes muertos que humillados"-; una suficiente distancia estratégica entre los actores relevantes para que puedan usar con eficacia su poder de amenaza en la negociación; y una predisposición de los actores al pacto mediante una visión a largo plazo de sus propios intereses y un criterio no-miope de elección (Colomer 1990, 1991a, 1991b, 1995a, 1995c; Colomer y Pascual., 1994).

Dentro de ese marco global, la relevancia que adquiere cada uno de los diferentes actores políticos -básicamente, los gobernantes "duros" y "blandos" y la oposición democrática- depende en parte de los condicionantes y las "exclusiones" impuestas por la situación inicial, en particular por el tipo de régimen autoritario. Pero, dentro de estos límites, la formación y la relevancia de los actores es también un resultado del propio proceso de cambio. De hecho, los actores pueden cambiar o adaptar sus preferencias a los resultados del proceso. Concretamente, los actores "maximalistas" pueden evolucionar hacia posiciones "gradualistas" con objeto de hacer viable la consecución de su alternativa preferida por medios pacíficos, si la oportunidad 
existe. También los actores "gradualistas" pueden modificar sus adhesiones con objeto de ganar mayor poder de negociación con sus interlocutores. Los diferentes actores pueden reforzarse mutuamente mediante la selección de sus interlocutores en las interacciones estratégicas, dado que la aparición pública de un actor en negociación con otros puede alimentar expectativas acerca de su fuerza futura e inducir a otras personas a darle su apoyo. En la relación general entre gobernantes y oposición, parece lógico esperar que el actor relativamente débil tratará de mantener la unidad en sus filas, pese a su probable diferenciación futura en varios partidos o tendencias ideológicas, mientras que el actor relativamente fuerte puede permitirse una anticipada diferenciación interna para asi poder negociar más ventajosamente el establecimiento de las futuras reglas del juego. En pocas palabras: los actores producen resultados, pero los resultados reales y esperados también inducen la formación y el crecimiento de los actores.

Desde esta perspectiva "estratégica", los valores culturales, las creencias morales y las actitudes psicológicas tienden a aparecer como elementos complementarios, fuertemente dependientes de las expectativas y las oportunidades de los actores, más que como factores causales básicos. Las percepciones de "honestidad" o "traición" en otros actores, el desprecio por la actitud de "medias-tintas" o, por el contrario, el elogio de la moderación, las ofertas magnánimas de reconciliación o la sed de venganza justiciera, así como la resolución de otros dilemas parecidos dependen fuertemente de la fuerza relativa de cada actor para imponer su voluntad sobre los demás, de las oportunidades efectivas de negociar y pactar y de la probabilidad de encontrarse implicado en un conflicto perjudicial y duradero. Cabe, pues, interpretar, que las decisiones de los actores guiadas por sus expectativas y sus cálculos estratégicos tienden a "seleccionar" sus correspondientes interlocutores, así como las actitudes y los valores apropiados, mediante las oportunidades de acuerdo y de éxito que las decisiones mismas crean.

\section{Incertidumbre del cambio y del régimen}

Un régimen democrático puede ser establecido mediante un pacto entre diferentes fuerzas políticas y sociales en la medida en que aparece como un acuerdo convencional acerca de unas nuevas reglas del juego, sin vinculación con ningún grupo particular de gobernantes o de políticas públicas.

En cambio, un régimen autoritario puede ser definido como aquel en el que unas reglas de decisión arbitrarias e injustas tienden a mantener a un número significativo de personas permanentemente excluidas de la oportunidad de elegir a los gobernantes. Es importante observar, no obstante, que para que un régimen autoritario que no sea meramente depredatorio (es decir, que no consista únicamente en el dominio de una banda de salteadores) pueda sobrevivir durante un período medianamente largo, la arbitrariedad y la exclusión deben ser compensadas con algún grado de satisfacción de los resultados, al menos con respecto a aquellas personas que le dan un apoyo decisivo o le prestan aquiescencia pasiva. En el caso particular de los regímenes de socialismo autoritario de la Unión Soviética y demás países de Europa oriental, la dictadura pudo durar porque los gobernantes se apoyaban en este tipo de intercambio con los súbditos excluidos de los derechos civiles y de la participación política: los gobernantes prometían bienestar material e igualdad social y los súbditos les correspondían con la renuncia a intervenir en los asuntos públicos.

Precisamente porque los regímenes autoritarios cuentan con una fuente de apoyo social basada en resultados sustantivos y no en procedimientos, pueden ser altamente vulnerables al incumplimiento de sus promesas o a la no-consecución de los resultados esperados. Esto es lo que ocurrió en particular con los regímenes de Europa oriental que habían fundado su legitimidad en un proyecto global de sociedad. Durante algún tiempo, los gobernantes comunistas sustituyeron los resultados reales en la realización de aquel proyecto por incentivos ideológicos orientados hacia el futuro (la promesa de un paraíso para las siguientes generaciones) y el terror disuasorio. Sin embargo, al cabo de un tiempo el fracaso en la consecución de la prosperidad y la igualdad prometidas apareció como ineludible. El escepticismo y el malestar se extendieron entre la población. A mediados de los años ochenta, el relevo de los máximos dirigentes por miembros de una nueva generación comportó un reconocimiento abierto de aquel fracaso global y abrió un nuevo proceso de cambio.

El incumplimiento de las promesas de un régimen autoritario puede tomar diversas formas. Puede consistir en una fuerte crisis económica, en la derrota militar ante un enemigo, o en una pérdida de fe de los súbditos en las creencias ideológicas en las que se hubieran fundado sus frustradas expectativas. La clave es que, ante el fracaso de los resultados, los súbditos descontentos tienden a reaccionar no sólo contra los gobernantes individuales sino también, dada la ausencia de reglas de decisión formales y previamente aceptadas para sustituirlos, contra la arbitrariedad y la inequidad de las reglas, es decir, contra el régimen. Cuando se trata de una dictadura personal en la que ni siquiera hay reglas para la sustitución del gobernante autoritario que hayan sido previamente aceptadas por quienes le han dado su apoyo, o bien tales reglas son impracticables, la mera derrota exterior 0 el fallecimiento del dictador pueden ser suficientes para hacer necesaria la adopción de nuevas reglas del juego.

A diferencia de estas características autoritarias, un régimen democrático es aquello en el que hay reglas de decisión 
conocidas y relativamente equitativas, las cuales producen una fuente procedimental y no sustantiva de apoyo social al régimen. Estas reglas incluyen los derechos civiles básicos de los ciudadanos y la elección competitiva de los gobernantes por los ciudadanos sobre una base relativamente igualitaria (es decir, por sufragio adulto amplio). Es bien cierto que las distintas fórmulas y ordenamientos institucionales (parlamentarismo o presidencialismo, sistemas electorales, descentralización, etc.) pueden ser evaluadas por su diferente capacidad de producir eficiencia y equidad de resultados. La responsabilidad de los gobernantes debe permitir o bien que el poder de decisión sea compartido por ciudadanos con distintos intereses y valores, o bien que esté en manos de coaliciones gobernantes homogéneas pero cambiantes, o ambas cosas. Pero todas las fórmulas deben permitir el relevo pacífico de los gobernantes y de las políticas públicas para que puedan ser consideradas democráticas.

No cabe, pues, esperar que el apoyo básico a la democracia proceda de sus buenos resultados o de la adhesión de los gobernantes a los "buenos" valores, sino que más bien son los resultados de la democracia los que son valorados como "buenos" porque son producidos a través de procedimientos previamente conocidos y equitativos. Esta fuente procedimental de apoyo social a la democracia la hace mucho menos vulnerable a las crisis económicas, las derrotas militares, el incumplimiento de las promesas de los gobernantes o la frustración de las expectativas de los ciudadanos que los regímenes autoritarios. En democracia, las decepciones y el descontento pueden ser canalizados hacia los gobernantes del momento y no contra las reglas básicas, las cuales permiten precisamente la sustitución de los gobernantes.

Ciertamente, muchas personas pueden preferir la democracia a la dictadura sobre la base de sus expectativas de alcanzar mayor bienestar material con un régimen democrático. Pero estas expectativas sólo pueden basarse en cruciales objetivos intermedios, los cuales resultan suficientes para la supervivencia de los regímenes democráticos. En democracia, en primer lugar, los ciudadanos tienen garantizados las libertades y los derechos civiles, los cuales incluyen la posibilidad de obtener información sobre los asuntos públicos y de discutir políticas públicas alternativas. En segundo lugar, los ciudadanos tienen la oportunidad de participar periódicamente en la selección de los gobernantes. Como consecuencia de todo ello, los ciudadanos pueden reaccionar a los eventuales fracasos colectivos o a los acontecimientos adversos de un modo más pacífico y ordenado que cuando se encuentran bajo unas reglas de decisión arbitrarias. Todas estas características pueden alimentar unas expectativas razonables de obtener resultados sociales, económicos y culturales favorables bajo un régimen democrático, pero también permiten la admisión de grados de satisfacción más bajos durante algunos períodos.
En comparación con la incertidumbre de las reglas autoritarias y la incertidumbre de los gobernantes y las políticas democráticas, la transición del autoritarismo a la democracia implica altos grados de incertidumbre a los dos niveles: las reglas y los gobernantes-políicas (para una discusión previa, véanse PRZEWORSKI, 1986: 58-59; MAINWARING et al., 1992: 312-317).

Hay que tener en cuenta que el punto de partida del proceso de cambio es la dictadura, un régimen caracterizado por la estabilidad de la coalición gobernante, dado que no se permite la alternancia en el gobierno. Bajo ese régimen, la toma de decisiones por los gobernantes está escasamente limitada y habitualmente conduce a la adopción de políticas públicas estables a medio plazo. Pero -como se ha dicho- sus reglas de decisión son arbitrarias e injustas, ya que todos aquellos cuyos valores o intereses resultan contradictorios con los de los gobernantes son permanentemente excluidos del gobierno y se encuentran en peligro de ser perseguidos por medios arbitrarios (como se suele decir, en una dictadura no sólo el lechero llama a la puerta de madrugada).

En comparación con esa situación inicial, es obvio que un proceso de transición implica incertidumbre de las reglas precisamente porque se trata de un proceso para cambiar las reglas arbitrarias existentes y el resultado -las nuevas reglas- es desconocido previamente. En lo que respecta a los gobernantes y las políticas de la transición, desempeñan un papel provisional, especialmente porque la principal "política pública" en ese período es precisamente la política institucional y de reforma, es decir, la sustitución del régimen anterior. Las expectativas de estabilizar los gobernantes y las otras políticas públicas de la transición dependen en gran medida del resultado institucional del proceso de cambio de régimen y de las oportunidades que éste ofrezca, por lo que son también muy inciertas.

Es esta incertidumbre general la que puede llevar a los actores a pactar unas nuevas reglas procedimentales que permitan mantener una incertidumbre permanente acerca de sus resultados políticos (quiénes serán los gobernantes y cuáles serán las políticas), pero que también permitan la supervivencia permanente de diferentes candidatos a gobernar con diferentes políticas, es decir, la democracia. La sustitución del autoritarismo por la democracia implica la sustitución de unos gobernantes y unas políticas ciertos bajo unas reglas arbitrarias y excluyentes por unos gobernantes y unas políticas inciertos bajo un esquema institucional básicamente estable.

La transición de la dictadura a la democracia comporta, por lo tanto, una creciente regulación de los procedimientos de decisión, los cuales se hacen más constrictivos, y una creciente incertidumbre acerca de los futuros gobernantes y políticas. Es esta doble faceta la que suele provocar sentimientos ambiguos durante la transición, ya que mientras algo nuevo, imprevisto y abierto se acerca, algo se cierra y las posibilidades que ofrecía el pasado quedan más circunscritas. 
Una vez en democracia, caben diversos grados de estabilidad de las políticas públicas y de equidad de las reglas; así, mientras que la unidad de poderes y el esquema "mayoritario" (a la británica) permiten drásticos giros en la orientación de los gobernantes y de las políticas mediante la alternancia de diferentes partidos en el gobiemo, la división de poderes y el pluralismo institucional y de partidos puede favorecer resultados bien de conflicto, bien de consenso mediante coaliciones amplias y políticas centristas. Pero en cualquier caso cierto grado de incertidumbre acerca de quiénes serán los gobernantes y que políticas ejecutarán es esencial en todos los regímenes democráticos.

La Tabla 1 resume la anterior discusión.

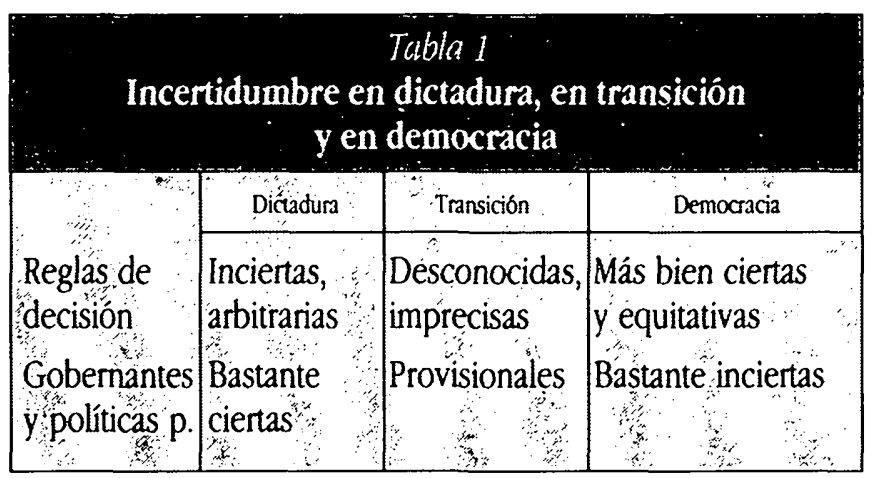

\section{Modelos de cambio y tipos de régimen democrático}

De acuerdo con la discusión arriba presentada, las relaciones entre tipos de régimen autoritario y tipos de régimen democrático no pueden establecerse en términos de relaciones directas entre "estructuras". El crucial proceso intermedio de transición es, por un lado, inducido por la situación inicial del régimen autoritario $y$, por el otro, conduce a un tipo particular de régimen democrático. Pero, como he subrayado, los procesos de transición se caracterizan por la relevancia que adquieren los actores y las decisiones estratégicas, cuyas decisiones e interacciones constituyen la variable básica para explicar la transformación estructural. En otras palabras: las diferentes formas de régimen autoritario presentan diferentes incentivos para la formación y la supervivencia de actores, pero son las decisiones de estos actores lo que produce el proceso que conduce a un nuevo tipo de régimen.

Si observamos, en primer lugar, las relaciones entre tipos de régimen autoritario y las diferentes vías de cambio que cabe definir mediante la relevancia de diferentes actores, parece posible establecer la "exclusión" de algunas hipótesis.
En primer lugar, un régimen autoritario muy restrictivo y excluyente -como el que existió en la Unión Soviética durante varias generaciones- difícilmente puede permitir la formación y supervivencia de movimientos de oposición, por lo que tiende a conceder un papel predominante a los gobernantes autoritarios tanto en la consolidación del régimen como en el comienzo de su cambio. Por ello, a partir de una situación inicial fuertemente autoritaria cabría descartar un proceso de cambio basado en una negociación formal entre los gobernantes "blandos" y la oposición -típicamente en torno a una "Mesa redonda".

En segundo lugar, y siguiendo un razonamiento análogo, tampoco parece razonable esperar un "colapso súbito" de un régimen autoritario -del tipo que se produjo en Checoslovaquia, Alemania oriental y Rumania a finales de 1989- si éste cuenta con un apreciable grado de pluralismo o "liberalización". Incluso si se produce un acontecimiento crítico inesperado, los gobernantes previamente evolucionados hacia posiciones "blandas" pueden reaccionar apropiadamente y tratar de preservar algunas posiciones de poder mediante una negociación con la oposición. Los regímenes autoritarios liberalizados son, por lo tanto, más propensos a inducir procesos negociados de transición -como los que tuvieron lugar en Polonia y HungríaPero incluso en esa situación inicial el papel de la oposición no puede darse por descontado, ya que unos gobernantes suficientemente hábiles pueden tratar de controlar las reformas desde arriba mediante transacciones entre las distintas facciones de los autoritarios y evitar los compromisos directos con la oposición.

Si observamos ahora las relaciones entre las vías de cambio y los tipos de régimen democrático, también cabe establecer algunas tendencias básicas. El primer modelo de cambio citado, basado en transacciones entre diferentes facciones de los gobernantes autoritarios (como en la URSS), permite a los gobernantes preservar significativos elementos de concentración de poderes y de continuidad institucional "mayoritaria", incluida la auto-transformación de los autoritarios en actores democráticos sin abandonar las posiciones de poder. En cambio, cuanto mayor es el poder de negociación de la oposición, más división de poderes y más innovación del régimen democrático hacia fórmulas institucionales pluralistas cabe esperar. Sin embargo, hay otras diferencias que dependen de la vía concreta de cambio que se haya seguido. La vía de negociación en una "Mesa redonda" promueve una política de reconciliación y de ausencia de represalias con respecto a los antiguos autoritarios que favorece el mantenimiento de prácticas consensuales en el contexto democrático. En cambio, el "colapso súbito" del régimen autoritario obliga a improvisar acuerdos más frágiles, habitualmente seguidos por una práctica de venganzas y purgas, que puede introducir mayores elementos de conflicto e inestabilidad en el régimen democrático resultante. (He desarrollado una 


\begin{tabular}{|l|l|l|}
\hline \multicolumn{3}{|c|}{ Tipos de régimen y modelos de transición } \\
\hline Régimen autoritario & \multicolumn{1}{|c|}{ Modelo de transición } & \multicolumn{1}{|c|}{ Régimen democrático } \\
\hline \multirow{4}{*}{ Dictadura } & $\begin{array}{l}\text { Colapso } \\
\text { súbito }\end{array}$ & $\begin{array}{l}\text { División de poderes: } \\
\text { pluralismo conflictivo }\end{array}$ \\
\cline { 2 - 3 } & $\begin{array}{l}\text { Transacción } \\
\text { entre autoritarios }\end{array}$ & $\begin{array}{l}\text { Unidad de poderes; } \\
\text { "Mayoritarismo" }\end{array}$ \\
\cline { 2 - 3 } & $\begin{array}{l}\text { Mesa redonda entre } \\
\text { gobntes."blandos"y } \\
\text { Libosición }\end{array}$ & $\begin{array}{l}\text { División de poderes; } \\
\text { pluralismo de } \\
\text { consenso }\end{array}$ \\
\hline
\end{tabular}

discusión más pormenorizada de estos aspectos en COLOMER 1995b, 1995d.)

La figura 1 resume de un modo esquemático estas relaciones entre tipos de régimen autoritario, modelos de transición y tipos de régimen democrático.

Este esquema puede ser sometido a contrastación con algunas características básicas de los procesos de democratización en Europa oriental antes aludidos.

Concretamente, en la mayoría de las antiguas Repúblicas de la Unión Soviética, posteriormente unidas en la Confederación de Estados Independientes, en las que la transición fue protagonizada por miembros o exmiembros recientes del Partido Comunista, la norma ha sido una alta concentración de poderes en manos de un Presidente con un reciente pasado comunista, un papel subordinado o simplemente inexistente del Parlamento, y un escaso protagonismo de los partidos políticos.

En cambio, en aquellos países en los que la transición a la democracia incluyó negociaciones formales entre los gobernantes y la oposición en torno a una "Mesa redonda" o una plataforma similar, pronto se han aceptado fórmulas en las que varios partidos comparten el poder o la alternancia en el gobierno. En concreto, los partidos excomunistas que habían evolucionado hacia partidos y politicas socialdemócratas moderados han vuelto al gobierno por vía electoral en Polonia, Hungría, Bulgaria y Lituania en 1993-94 (si en Estonia y Lituania no se ha producido este giro ha sido debido a la política de los nuevos gobernantes de negar el derecho de voto a la mayoría de los habitantes noindigenas, en su mayor parte de origen ruso o eslavo).

Por último, la inestabilidad y el conflicto fueron muy altos en los primeros años de los regímenes democráticos estableci- dos en aquellos países que experimentaron un colapso súbito del régimen autoritario. Mientras que la Alemania oriental fue absorbida por la República Federal de Alemania, Checoslovaquia se escindió en dos Repúblicas separadas y Rumania ha sufrido frecuentes turbulencias y un cuestionamiento general de su condición democrática.

Esta variedad de resultados institucionales y tipos de régimen democrático no significa necesariamente que la democracia no pueda considerarse "consolidada" en muchos de los países mencionados. Si la "consolidación" de la democracia se relaciona con la "calidad" de sus resultados, es decir, con su capacidad de producir decisiones, leyes, políticas públicas y actos administrativos eficientes y satisfactorios para amplios sectores de la ciudadanía, es evidente que los diferentes esquemas institucionales y sistemas de partidos - producidos a su vez por diferentes vías "estratégicas" de cambio- introducen diferentes sesgos y producen diversos grados de ineficiencia colectiva en la toma de decisiones. Desde este punto de vista, cabría decir que ningún régimen democrático está nunca "consolidado" del todo, dado que siempre hay sectores de ciudadanos más o menos significativos que pueden desear y apoyar nuevas propuestas de reforma del régimen. Mejorar la "calidad" de los resultados de la democracia es, en este sentido, una tarea que nunca termina.

Pero los deseos de los ciudadanos tienden a formarse también en respuesta a los incentivos ofrecidos por el esquema institucional existente y a las decisiones colectivas, no sólo de un modo reactivo sino también adaptativo. Como consecuencia de ello, y a pesar de sus diferentes grados de ineficiencia y equidad, los regímenes democráticos tienen poderosos mecanismos auto-estabilizadores en comparación con los regímenes autoritarios. En este sentido de estabilidad del régimen, cabe considerar que la democracia se encuentra "consolidada" en todas aquellas situaciones en las que ningún actor tiene suficiente poder de negociación para emprender unilateralmente un nuevo proceso de cambio político. Pueden tener lugar entonces nuevos acontecimientos críticos y fracasos colectivos, como una recesión económica, una secesión territorial o un nuevo conflicto militar - tal como ha ocurrido en Europa oriental en los años noventa-. Pero estos acontecimientos, que podrían haber resultado fatales para la supervivencia de los regímenes autoritarios, pueden ser absorbidos por instituciones democráticas relativamente estables que permitan un relevo pacífico de los gobernantes. Este parece haber sido también el caso en muchos de los nuevos Estados democráticos de Europa oriental. 


\section{Bibliografia}

COLOAER, Josep M. (1990): El arte de la manipulación política. Votaciones y teoria de juegos en la politica española. Barcelona: Anagrama.

- 1991a. "Transitions by Agreement. Modeling the Spanish Way". American Political Science Review, 85, 4; 1283-1302.

- 119lb. "La vía báltica", Claves de Razón Práctica, 18.

- 1994. "Teorias de la transición", Revista de Estudios Políticos, 86: 243-253.

- 1995a. Game Theory and the Transition to Democracy. The Spanisb Model. Hampshire: Edward Elgar.

- 1995b. "Strategies and Outcomes in Eastern Europe", Joumal of Democracy, 6, 2: 74-85 (urad. "Estrategias institucionales en Europa oriental", Claves de Razón Práctica, 49).

- 1995c. "La vía cubana a la democracia", Claves de Razón Práctica, 31.

- 1995d. "Reprisals or Reconciliation in Transitions to Democracy". Constitutional Reform in Eastern Europe, Oiono (irad. "Venganza democrática o reconciliación", Claves de Razón Práctica, 58).
Colomer, Josep M., y Margot P.scainl. (1994): "The Polish Games of Transition", Communist and Past-Communist Studies, 27, 3: 275-294.

LIPSET, Seymour; M. Kyoung-Ryung SeONG, y Johyn Charles ToRRes (1993): "Análisis comparado de los requisitos sociales de la democracia", Revista Internacional de Ciencias Sociales, 136: 175-198.

Manwaring; SCotT; Guillermo O'Donvell, y J. Samuel Valenzuela eds.(1992) Issues in Democratic Consolidation. Democracies in Comparative Perspective. Notre Dame: University of Notre Dame Press.

Przeworski, Adam. 1986. "Some Problems in the Study of the Transition to Democracy", en Guillermo O'Donnell, Philippe Schmitter y Laurence Whitehead eds. Transitions from Autboritarian Rule: Prospects for Democracy. Baltimore: Johns Hopkins University Press (Irad. Transiciones desde un régimen autoritario. Buenos Aires: Paidós, 1989). 


\section{Ef}

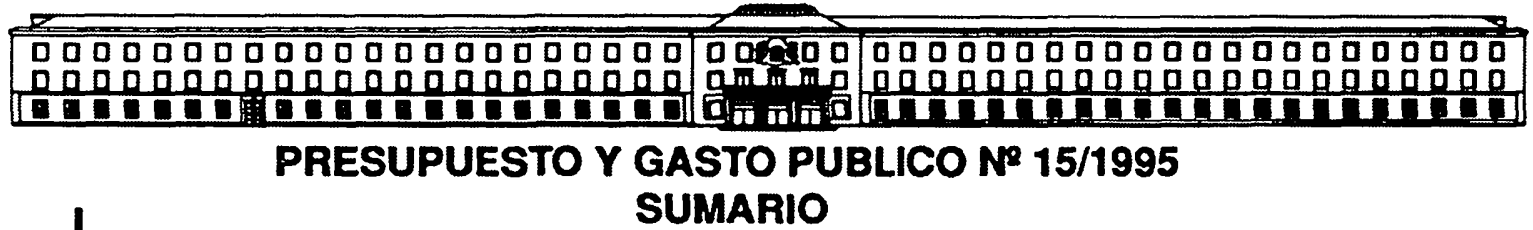

\section{Edtorial}

COLABORACIONES

Anállsis del porcentaje de la penelofich jubllación del Régimen General de la Seguridad

Soclal. Estimacion Técnica

Antonio Redecillas Lopez de Stituling.

Actuario

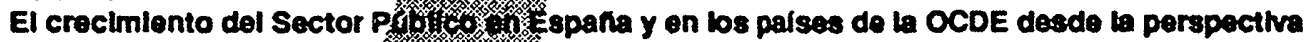
del empleo público

Germán Carrasoo Castillo

José M. Dominguez Martiná,

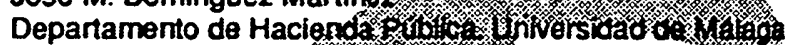

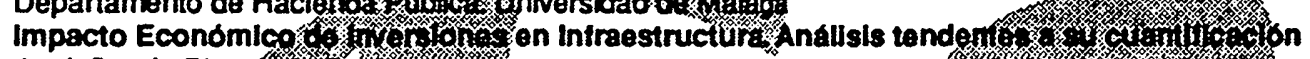

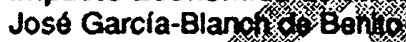

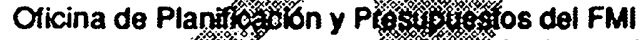

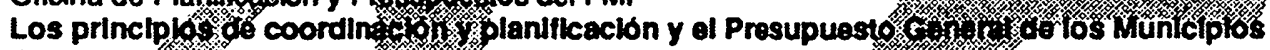

Gloria Alarosingarcia

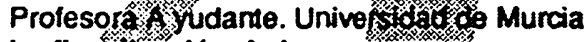

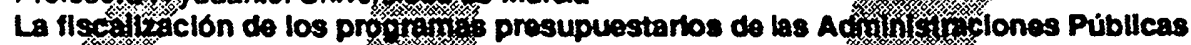

Lourdasiontos Pradas

Proflestra Titular. Universid ha d d Garagoza

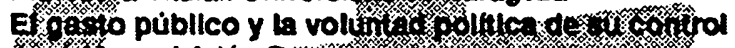

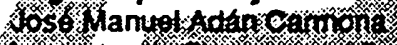

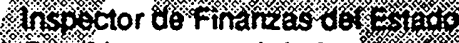

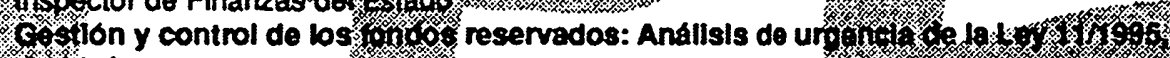
ofo 1 de mayo

inose Pascual Garcia

Doctor en Derecho. Inspe

\section{SEMINARIO SOBRE OhIESENTE Y FUTURO DEL}

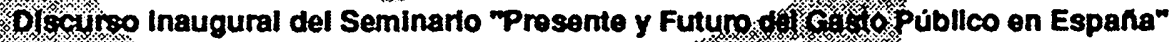

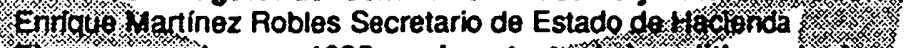

El presupuesto para 1995 en el contexto of a th porifica actial

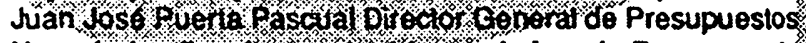

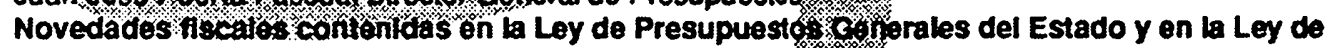
Acompanamlento

Eduardo Abril Abadin Director General de Tributos

El reto de la Convergencla y las perspectivas financleras coni i union Europea

Angel Torres Torres Director General de Planificación

Medidas de control det Déflct Público

Federico Montero Hita

Secretario General de Planiticación y Presupuestos

El futuro en el control del Gasto Público

Santiago Fuentes Vega Subdirector General de Estudios y Coordinacion

Una polftica presupuestaria para alcanzar el Déflctt de Comvergencla en 1999

Carlos Monasterio Escudero Universidad de Oviedo

Discurso de clausura del Seminario "Presente y Futuro del Gasto Público en Espana"

Pedro Solbes Ministro de Economía y Hacienda

Instituto de Estudios Fiscales

Ministerio de Economía y Hacienda

Secretaría de Estado de Hacienda

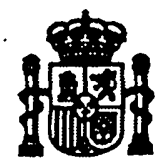

